\title{
Merlin in organ size control and tumorigenesis: Hippo versus EGFR?
}

\author{
Chunling Yi and Joseph L. Kissil ${ }^{1}$ \\ Molecular and Cellular Oncogenesis Program, The Wistar Institute, Philadelphia, Pennsylvania 19104, USA
}

The role of the NF2 gene as a tumor suppressor has been well established. In this issue of Genes \& Development, Benhamouche and colleagues (pp. 1718-1730) demonstrate that NF2 is also involved in the regulation of organ size control in mammals. Conditional knockout of $N f 2$ in the mouse liver results in massive organ enlargement and eventual tumor development, which is attributed to the specific expansion of oval cells. Here we discuss these findings and the proposed molecular mechanisms involved within the context of our current understanding of the pathways regulated by NF2.

\section{Background}

Neurofibromatosis type 2 (NF2) is an inherited cancer syndrome that is characterized by the development of schwannomas and meningiomas. With the identification of the NF2 gene nearly two decades ago, the door was opened for the study of the encoded gene product Merlin. Research from numerous groups in the subsequent years would bring to light some of Merlin's functions. However, the emerging picture was one of significant complexity: Merlin was associated with multiple functions that appeared to be dependent on spatial and temporal constraints. Within a few years following the initial gene discovery, animal models involving the $N f 2$ allele were developed that greatly facilitated the understanding of Merlin's function as a tumor suppressor, and elucidated a critical role for $N f 2$ in development (McClatchey et al. 1997, 1998).

The first mouse model of NF2 sought to mimic the heterozygous status observed in NF2 patients by generating animals with a single knockout allele of $\mathrm{Nf2}$ (McClatchey et al. 1998). Like NF2 patients, $\mathrm{Nf2}^{+/-}$mice were predisposed to develop cancer. However, the spectrum of tumors that arose in these mice was very different from that of NF2 patients. While NF2 patients develop primarily schwannomas and meningiomas, the $\mathrm{Nf2}^{+/-}$mice acquired osteosarcomas, fibrosarcomas, and

[Keywords: EGFR; Merlin; NF2; cholangiocellular carcinoma; hepatocellular carcinoma; liver progenitor]

${ }^{1}$ Corresponding author.

E-MAIL jkissil@wistar.org; FAX (215) 898-3874.

Article is online at http://www.genesdev.org/cgi/doi/10.1101/gad.1964810. hepatocellular carcinomas (HCCs). Importantly, all these tumors exhibited loss of the wild-type $N f 2$ allele, indicating that loss of both functional alleles of $N f 2$ was a prerequisite for tumor formation. The reasons for the discrepancies between patients and the mouse model are not clear, but one possible explanation could be differences in the rate of loss of heterozygosity $(\mathrm{LOH})$ of the remaining wild-type allele. Indeed, further refinement of the model to a spatially controlled system where both $N f 2$ alleles were knocked out by a Cre recombinase driven by the P0 promoter $(\mathrm{PO}-\mathrm{Cre})$ - which is expressed specifically in myelinating Schwann cells-resulted in Schwann cell hyperplasia and schwannoma development, recapitulating some of the manifestations of the human disease (Giovannini et al. 2000).

In addition to the use of mouse models, the function of Merlin has been studied extensively in flies and mammalian cell culture systems. Numerous Merlin-interacting proteins have been identified through various approaches, and have linked Merlin to a number of signaling pathways and cellular functions (Scoles 2008). Figure 1 outlines two major aspects of Merlin growth-suppressive functions that have come to light from these studies: (1) negative regulation of receptor tyrosine kinase (RTK) signaling and downstream effectors, and (2) upstream modulation of the Hippo pathway. Merlin negatively regulates RTK signaling by either directly controlling the turnover of RTKs from the cell membrane or interfering with the activation of signaling components downstream from RTKs (Curto and McClatchey 2008). Previous studies in flies and more recent studies in mammalian cells suggest that Merlin acts as an upstream regulator of the Hippo pathway, a conserved tumorsuppressive pathway that plays important roles in restricting organ size and suppressing tumor development in both flies and mammals (Zhao et al. 2010).

\section{Organ size control}

Mutations in Merlin (Mer) and its related protein, Expanded (Ex), cause pronounced tissue outgrowth in multiple organs in Drosophila melanogaster, phenocopying mutations of Hippo pathway components (Hamaratoglu et al. 2006). The core Hippo pathway is composed of a kinase cascade, in which the Hippo (Hpo) kinase, in complex with the scaffold protein Salvador (Sav), phosphorylates Warts (Wts) and 


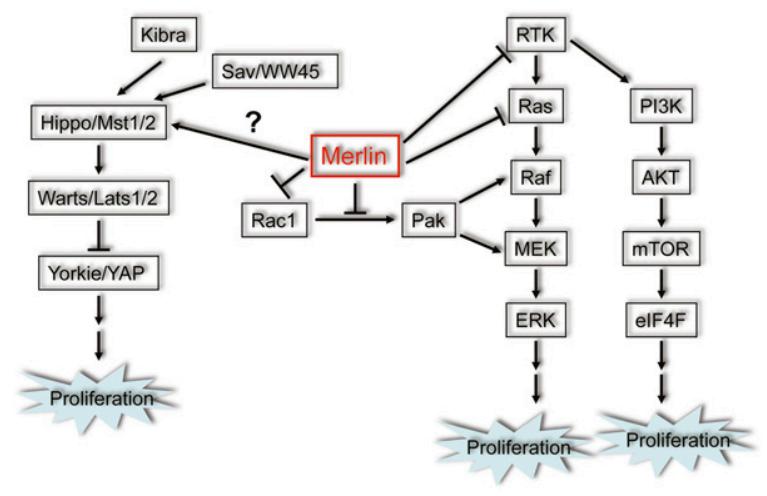

Figure 1. Schematic representation of currently identified points of interaction between Merlin and the Hpo/Yap and Ras/Rac signaling pathways discussed here. Merlin has been shown to function as a regulator of additional signaling mechanisms; however, these interactions were omitted for clarity.

Mats. Phosphorylated Mats binds to and further activates the Wts kinase, which in turn phosphorylates Yorkie (Yki), a transcriptional coactivator. The phosphorylation of Yorkie prevents it from entering into the nucleus, where it forms transcriptionally active complexes with Scalloped (Sd) and other transcription factors to drive the expression of proproliferative or anti-apoptotic genes (Zhao et al. 2010). Epistasis studies have placed Mer and Ex upstream of Hpo and Wts, and overexpression of Mer/ Ex causes Wts hyperphosphorylation and inhibits the function of Yki as a transcription activator (Hamaratoglu et al. 2006). An alternative theory of how Mer/Ex regulates cell proliferation has emerged from another study focusing on the Drosophila eye development (Maitra et al. 2006). In this study, Maitra et al. (2006) demonstrated that loss of mer/ex leads to trafficking defects and subsequent increase in the signaling output of several transmembrane receptors such as EGFR and Notch, which could also explain the overgrowth phenotypes observed in mer/ex mutant cells. Thus, it remains an open question which of the two mechanisms is dominant in mediating the growth control function of Mer/Ex.

In this issue of Genes \& Development, elegant work from the McClatchey group (Benhamouche et al. 2010) demonstrates for the first time that mammalian Merlin, similar to its Drosophila counterpart, is also involved in tissue size control-specifically, in the liver. By generating mice with Cre recombinase under the control of the albumin promoter (Alb-Cre) and conditional knockout alleles of $N f 2\left(N f 2^{\text {lox/lox }}\right)$, Benhamouche et al. (2010) were able to delete $N f 2$ in hepatoblasts (HBs) starting at embryonic day 9.5 (E9.5). In all of the Alb-Cre: Nf2 $2^{\text {lox/lox }}$ mice, this led to massive enlargement of the liver, which was attributed to the specific expansion of oval cells (OCs), the facultative bipotential liver progenitor cells with characteristically small cell size and large ovoid nuclei that normally first appear in the periportal region and then infiltrate along the bile canaliculi.

To gain an understanding of the molecular mechanisms underlying the distinctive phenotype caused by Nf2 loss in the liver, Benhamouche et al. (2010) examined the Hippo pathway, which is well conserved in mammals and has been implicated recently in liver size control and liver cancer (Camargo et al. 2007; Dong et al. 2007; Zhou et al. 2009; Lee et al. 2010; Lu et al. 2010; Song et al. 2010) and EGFR signaling, which was connected to Merlin's tumor-suppressive activities by previous work from the McClatchey laboratory (Curto et al. 2007; Cole et al. 2008; Morris and McClatchey 2009).

\section{The Hippo connection?}

Existing evidence suggests that Merlin also participates in Hippo signaling in mammalian cells. Merlin has been shown to regulate the expression and localization of Yap (Yki homolog) in schwannoma cells, and knockdown of Yap rescues the hyperproliferative phenotype of NF2deficient meningioma and mesothelioma cells (Zhao et al. 2007; Striedinger et al. 2008; Yokoyama et al. 2008). A recent study demonstrated that Merlin acts synergistically with a newly identified Hippo pathway component, Kibra, to promote Lats1/2 (homologs of Wts) phosphorylation (Yu et al. 2010).

A series of recent studies has shown that the mammalian Hippo pathway also regulates organ size, particularly of the liver, akin to what has been observed in flies. Inducible overexpression of Yap in the adult mouse liver results in rapid increase in liver size, which can be reversed upon withdrawal of doxycycline (Table 1; Camargo et al. 2007; Dong et al. 2007). Comparable hepatomegaly phenotypes were observed when Mst1 and Mst2 (homologs of $\mathrm{Hpo}$ ) were ablated specifically in the liver during embryonic development with $A$ lb-Cre, or neonatally using a CAGGCre-ER system (in which the expression of a tamoxifen-dependent form of Cre recombinase is driven by the chicken $\beta$-actin promoter/enhancer to express in a broad spectrum of tissues), or in adult mice through $A d$ Cre injection (Table 1; Zhou et al. 2009; Lu et al. 2010; Song et al. 2010). Likewise, Alb-Cre-mediated liverspecific knockout or CAGGCre-ER-induced compound deletion of WW45 (Sav homolog), an adaptor for Mst1/2, also gives rise to liver enlargement (Table 1; Lee et al. 2010; Lu et al. 2010).

Given the similar phenotypes produced by deletions of Nf2 and Hippo pathway components using the same conditional Cre system (Table 1), and previous studies in both fly and mammalian cells linking Merlin to the Hippo pathway, Benhamouche et al. (2010) sought to assess whether loss of $N f 2$ in the liver leads to deregulation of Hippo signaling. Surprisingly, they did not find evidence of further Yap activation in cultured $\mathrm{Nf2} 2^{-/-}$ OCs, nor did the knockdown of Yap in these cells rescue their hyperproliferative phenotypes. Based on these findings, Benhamouche et al. (2010) conclude that Merlin regulates organ size through a mechanism independent of the Hippo pathway.

One remarkable feature of $N f 2$ deletion in the liver is that it causes specific expansion of OCs without apparently affecting surrounding hepatocytes (Table 1). Since previous studies manipulating Mst1/2 or Yap expression 
Table 1. Comparison of liver phenotypes between the Alb-Cre:Nf2 ${ }^{\text {lox} / \mathrm{lox}}$ mice and various mouse models with knockout or transgenic expression of Hippo or EGFR pathway components

\begin{tabular}{|c|c|c|c|c|c|c|}
\hline Gene names & $\begin{array}{c}\text { Cre or promoter } \\
\text { alleles }\end{array}$ & $\begin{array}{l}\text { Change in } \\
\text { liver size }\end{array}$ & $\begin{array}{c}\text { OC } \\
\text { expansion }\end{array}$ & $\begin{array}{l}\text { Tumor } \\
\text { types }\end{array}$ & $\begin{array}{l}\text { Change in Yap } \\
\text { phosphorylation }\end{array}$ & $\begin{array}{c}\text { Yap protein } \\
\text { accumulation }\end{array}$ \\
\hline \multirow[t]{3}{*}{$N f 2$} & Alb-Cre & Yes & Yes & $\mathrm{HCC} / \mathrm{CC}$ & No & Yes \\
\hline & Ad-Cre injec. $+P H x$ & No & Yes & $\mathrm{HCC} / \mathrm{CC}$ & No & Yes \\
\hline & Mx1-Cre + PHx & No & Yes & $\mathrm{HCC} / \mathrm{CC}$ & No & Yes \\
\hline \multirow[t]{4}{*}{ Mst1/2 } & Ad-Cre injec. & Yes & $\mathrm{N} / \mathrm{E}$ & HCC & Yes & Yes \\
\hline & Alb-Cre & Yes & $\mathrm{N} / \mathrm{E}$ & HCC & Yes & Yes \\
\hline & CAGGCre-ER & Yes & Yes & $\mathrm{HCC} / \mathrm{CC}$ & Yes & Yes \\
\hline & MMTV-Cre & Yes & $\mathrm{N} / \mathrm{E}$ & HCC & Yes & Yes \\
\hline \multirow[t]{3}{*}{ WW45 } & Alb-Cre & Yes & Yes & $\mathrm{HCC} / \mathrm{CC}$ & No & Yes \\
\hline & MMTV-Cre & Yes & $\mathrm{N} / \mathrm{E}$ & $\mathrm{HCC} / \mathrm{CC}$ & $\mathrm{N} / \mathrm{E}$ & $\mathrm{N} / \mathrm{E}$ \\
\hline & CAGGCre-ER & Yes & $\mathrm{N} / \mathrm{E}$ & $\mathrm{HCC} / \mathrm{CC}$ & $\mathrm{N} / \mathrm{E}$ & $\mathrm{N} / \mathrm{E}$ \\
\hline \multirow[t]{2}{*}{ YAP } & ApoE/tTA & Yes & $\mathrm{N} / \mathrm{E}$ & HCC & $\mathrm{N} / \mathrm{A}$ & N/A \\
\hline & $L A P / t T A$ & Yes & $\mathrm{N} / \mathrm{E}$ & $\mathrm{N} / \mathrm{E}$ & $\mathrm{N} / \mathrm{A}$ & $\mathrm{N} / \mathrm{A}$ \\
\hline EGFR & Alb-Cre & No & $\mathrm{N} / \mathrm{A}$ & $\mathrm{N} / \mathrm{A}$ & $\mathrm{N} / \mathrm{E}$ & $\mathrm{N} / \mathrm{E}$ \\
\hline \multirow[t]{2}{*}{$T G F \alpha$} & MT1/zinc & Yes & $\mathrm{N} / \mathrm{E}$ & $\mathrm{HCC}$ & $\mathrm{N} / \mathrm{E}$ & $\mathrm{N} / \mathrm{E}$ \\
\hline & Elastase & Yes & $\mathrm{N} / \mathrm{E}$ & $\mathrm{HCC}$ & $\mathrm{N} / \mathrm{E}$ & $\mathrm{N} / \mathrm{E}$ \\
\hline
\end{tabular}

(N/E) Not examined; (N/A) not applicable; (PHx) partial hepatectomy; (injec.) injection.

in the adult liver led to overproliferation of hepatocytes (Table 1; Camargo et al. 2007; Dong et al. 2007; Zhou et al. 2009), Benhamouche et al. (2010) hypothesize that Merlin and the Hippo pathway may operate in distinct cell types within the liver. However, more recent studies indicate that liver-specific knockout of other elements of the Hippo pathway-namely, WW45 and Mst1/2—during development also results in OC expansion, to various degrees (Table 1; Lee et al. 2010; Lu et al. 2010). Given these findings, it is evident that further studies are needed to clarify the picture. For example, in the earlier reports, the effects of Yap overexpression on liver development were not addressed (Camargo et al. 2007; Dong et al. 2007). In addition, conditional deletions of Lats $1 / 2$ in the liver are yet to be reported. Perhaps activating Yap or inactivating Lats1/2 during development (by crossing either conditional Yap transgenic or conditional Lats1/2 knockout mice with $A 1 b$-Cre) and probing for the cell(s) of origin that give rise to the apparent tissue expansion will help shed light on whether or not the OC phenotypes are restricted to a subset of the Hippo pathway components, as current studies suggest.

Despite their similarities, one major phenotypic difference distinguishes loss of $N f 2$ from that of Mst1/2 and Yap. Unlike Mst1/2 and Yap, Nf2 loss alone-either via Ad-Cre injection or by crossing with Mx1-Cre-is insufficient to drive liver expansion in adult mice. Only when $N f 2$ deletion is combined with liver injury induced by partial hepatectomy ( $\mathrm{PHx})$ do OC expansion and subsequent liver enlargement occur, consistent with Merlin playing a more limited or distinctive role in maintaining tissue quiescence. Interestingly, reminiscent of $N f 2$ knockouts, abrogation of WW45 does not appear to affect Lats or Yap phosphorylation (Lee et al. 2010; Lu et al. 2010). One study has suggested that WW45 functions by regulating the expression level of core Hippo pathway components (Lee et al. 2010). Notably, loss of Merlin in OCs also seems to cause accumulation of Lats and Yap proteins (Supplemental Figs. 3, 4; Benhamouche et al.
2010). Recently, Sav, the fly ortholog of WW45, has been shown to interact directly with the FERM domain of Merlin through a conserved FERM-binding motif (FBM) (Yu et al. 2010). Therefore, it is plausible that a putative Merlin/WW45 complex may engage in additional functions that do not involve an Mst/Lats/Yap phosphorylation cascade in OCs.

\section{The EGFR twist?}

Based on their previous findings, and the apparent lack of further Yap activation in Nf2-null OCs, Benhamouche et al. (2010) propose that the OC expansion phenotype observed in Nf2 liver mouse models might be mediated through Merlin's regulation of EGFR signaling. The McClatchey group (Curto et al. 2007; Cole et al. 2008; Morris and McClatchey 2009) has shown previously that Merlin regulates the internalization and signaling of EGFR in response to the formation of cell:cell contacts. To investigate this hypothesis, Alb-Cre:Nf2 $2^{\text {lox/lox }}$ mice were treated with erlotinib, a tyrosine kinase inhibitor with high specificity toward EGFR. This treatment resulted in a dramatic decrease in lesion size and in liver: body weight ratio, which were accompanied by reduced cellular proliferation rates and EGFR signaling outputs. These findings suggest that EGFR activity is likely required for OC expansion in the Nf2-null livers.

EGFR signaling has been implicated previously in regulation of liver growth in mouse models. A recent study showed that Alb-Cre-driven perinatal deletion of Egfr did not reduce liver size (Table 1), although it did lead to impaired liver regeneration after PHx (Natarajan et al. 2007). Furthermore, in vivo infusion of EGFR ligands has been shown to enhance chemical-induced OC proliferation (Nagy et al. 1996). Direct proof that EGFR can indeed drive OC proliferation and liver hyperplasia will require generation of liver-specific EGFR transgenic mouse models, which, to our knowledge, have not yet been reported. 
Interestingly, an earlier study demonstrated that ubiquitous overexpression of TGF $\alpha$, an EGFR ligand, in neonatal MT1-TGF $\alpha$ transgenic mice results in enlargement of the liver as well as several other organs (Table 1; Sandgren et al. 1990). The kidney and limbs do not appear to be enlarged in MT1-TGF $\alpha$ transgenic mice, despite the presence of high levels of TGF $\alpha$ in these organs. Notably, similar spectrums of affected and unaffected organs were observed when Mst1/2 were silenced at birth (Song et al. 2010), suggesting a possible link between EGFR and Hippo signaling.

\section{Signaling cross-talk?}

Loss of Mer and Ex in Drosophila causes down-regulation of the Hippo pathway, as well as accumulation of transmembrane receptors such as EGFR (Hamaratoglu et al. 2006; Maitra et al. 2006). Similarly, in mammalian cells, Merlin has been shown to regulate both Hippo and EGFR signaling (Curto et al. 2007; Cole et al. 2008; Striedinger et al. 2008; Yokoyama et al. 2008; Morris and McClatchey 2009; Yu et al. 2010). Considering that perturbing Hippo or EGFR signaling in mouse models gives rise to very similar overgrowth phenotypes in a subset of tissues, it is quite likely that these phenotypic overlaps are more than coincidental and that, likely, the Hippo and EGFR signaling pathways are closely interlinked.

Indeed, cross-talk between downstream components of EGFR signaling and the Hippo pathway has been reported. In particular, Raf-1 has been shown to bind directly to Mst2 and prevent Mst2 from associating with RASSF1A, which, upon forming a complex with Mst2, stimulates its kinase activity and enhances its interaction with Lats1 (O'Neill et al. 2004; Matallanas et al. 2007). Interestingly, two common Akt phosphorylation sites have been identified recently on Mst1 and Mst2 (Jang et al. 2007; Romano et al. 2010; Yuan et al. 2010). At least in the case of Mst2, phosphorylation of these sites by Akt in response to mitogenic stimuli disrupts Mst2RASSF1A association, while promoting the formation of the Mst2-Raf-1 inhibitory complex (Romano et al. 2010). Taken together, these studies suggest that EGFR, through two downstream effector pathways (namely, the MAPK and $\mathrm{PI} 3 \mathrm{~K}$ pathways), could indirectly control the activity of $M s t 1 / 2$, the currently identified most upstream kinases of the Hippo signaling cascade.

On the other hand, there is also evidence of modulation of EGFR signaling by the Hippo pathway. A recent study identified the EGFR ligand amphiregulin (AREG) as a transcriptional target of Yap, and Zhang et al. (2009) nicely demonstrated that Yap-induced expression and secretion of AREG up-regulates EGFR signaling of neighboring cells through a non-cell-autonomous mechanism. Similarly, ectopic expression of $\mathrm{YAP}^{\mathrm{S} 127 \mathrm{~A}}$, the constitutively active form of human YAP in Drosophila, specifically induced the expression of the EGFR ligand Vein, leading to an increase in EGFR signaling. The intertwined nature of the relationship between the EGFR and Hippo pathways was corroborated further by genetic studies in flies: The overgrowth phenotype caused by increased activity of yki can be partially suppressed by loss-of-function mutants of egfr or vein, whereas reduced Yki activity resulting from overexpression of its negative regulators, Wts and Hpo, is enhanced by egfr or vein mutants. Interestingly, EGFR ligands including AREG are mitogenic to mouse OCs in culture, and AREG mediates protection against liver injury in a mouse model (Berasain et al. $2005 a, b)$. Therefore, it would be interesting to compare the expression levels of EGFR ligands in Nf2-null livers with those in wild-type livers. This might help explain the elevated levels of EGFR signaling in Nf2-null OCs, and the beneficial effect of Erlotinib treatment toward tumors caused by loss of $N f 2$.

\section{Liver tumorigenesis}

\section{Mouse models}

Benhamouche et al. (2010) report that, after the initial OC expansion, the Alb-Cre:Nf2 ${ }^{l o x / l o x}$ mice invariably develop liver cancer. This is consistent with their previous findings in $\mathrm{Nf2}^{+/-}$mice (McClatchey et al. 1998). A unique aspect of liver cancer in the Nf2 conditional knockout mouse models is the development of both HCC of various histopathological types and cholangiocarcinoma (CC) lesions that resemble a poorly differentiated subtype thought to originate from the putative location of an adult liver stem cell niche (Table 1).

Similarly, both HCC and CC were found in Mst1/2 and WW45 conditional knockout mice, and tumor development in these mice was preceded by massive OC expansion in the liver (Table 1; Lee et al. 2010; Lu et al. 2010; Song et al. 2010). Only HCC was reported in the two previous Yap transgenic studies (Camargo et al. 2007; Dong et al. 2007). However, in both cases, the Yap transgene was activated only in adult mice. An interesting question is whether turning on Yap at an earlier time point will alter the tumor spectrum in the liver. Notably, when $M s t 1 / 2$ were inactivated during adulthood, as in the case of $M s t 1^{-1-}: M s t 2^{+/-}$and Mst1/2 conditional mice injected with Ad-Cre, HCC primarily occurred (Table 1; Zhou et al. 2009). In contrast, when Nf2 was deleted from adult mouse livers in the present study, tumors of both HCC and CC types arose only after OC proliferation was stimulated by PHx (Table 1). Therefore, unlike Mst1/2 and Yap, which appear to function in both hepatocytes and OCs, Merlin's function in the liver appears to be restricted to OCs.

As mentioned earlier, liver-specific Egfr transgenic mouse models have not been described. However, transgenic mice overexpressing TGF $\alpha$ in the liver have been shown to develop HCC (Table 1; Jhappan et al. 1990; Sandgren et al. 1990). Interestingly, a recent wholegenome transposon-based insertional mutagenesis screen for HCC-associated genes in mice identified transposon insertions in the Egfr gene in $85 \%$ of preneoplastic liver nodules (Keng et al. 2009). The most frequent insertion site within the Egfr gene was predicted to result in a truncated activated form of EGFR. When this mutated allele of EGFR was expressed in the liver, it led to tumor 
development, implying that EGFR can function as a driver of HCC in mice.

\section{Implications for human disease}

Homozygous NF2 mutations have been reported previously in two human HCC cell lines (Pineau et al. 2003). However, NF2 mutations have not yet been linked to human CC. The unique mouse models of combined HCC and CC generated by Benhamouche et al. (2010) point toward a potential role of Merlin in maintaining the quiescence of liver progenitor cells. Hopefully, this pioneering study will elicit an interest in the analysis of Merlin/NF2 status in human liver cancer, particularly in tumors that bear progenitor cell-of-origin signatures, which should help elucidate the potential role of Merlin/ NF2 in human liver cancer.

EGFR has long been implicated in human liver cancers. An estimated $40 \%-70 \%$ of HCCs overexpress EGFR (Ito et al. 2001a; Buckley et al. 2008). Overexpression or mutation of EGFR is also a frequent event associated with human CCs (Ito et al. 2001b; Leone et al. 2006). Recently, therapies targeting EGFR with Erlotinib in combination with the VEGF inhibitor Bevacizumab have shown efficacy toward both HCC and CC in phase II clinical trials (Thomas et al. 2009; Lubner et al. 2010).

The deregulation of Hippo signaling as a potential major driving force for human HCCs has only recently become appreciated. Remarkably, several recent studies demonstrated that elevated expression, reduced S127 phosphorylation, and/or increased nuclear localization of Yap are present in 50\%-60\% of human HCC specimens (Zender et al. 2006; Xu et al. 2009; Zhou et al. 2009). Furthermore, decreased Yap phosphorylation is closely associated with reduced levels of p-Mob (Mats ortholog), a direct substrate of Mst1/2 and a coactivator of Lats1/2 (Zhou et al. 2009). Consistent with lowered Mst1/2 activities, $60 \%$ of human HCC samples examined lack the cleaved and constitutively active forms of Mst1/2 (Zhou et al. 2009). It is worth noting that an increase in Yap protein levels and nuclear staining was also observed in a subset of tumors in Alb-Cre: Nf2 $2^{\text {lox/lox }}$ mice (Supplemental Fig. 8; Benhamouche et al. 2010), suggesting a role for Hippo pathway deregulation at least during the late stages of tumor development. Interestingly, Yap overexpression in HCC is associated with poorer tumor differentiation, a higher rate of tumor recurrence after hepatectomy, and lower overall survival rates (Xu et al. 2009).

Given the high percentage of EGFR and Yap activation in human HCCs, their phenotypic resemblance in mouse models, and evidence of cross-talk between EGFR and Hippo signaling, a comparative analysis of EGFR and Yap activities within the same patient samples should yield important clues to the relationship between EGFR and Hippo signaling in mediating liver cancer development.

Finally, in addition to liver cancer, both EGFR and the Hippo pathway components have been implicated in several other types of cancer (Lurje and Lenz 2009; Zhao et al. 2010). Previously underappreciated roles of Merlin in common types of sporadic cancers that are not associated with NF2 have also come to light recently (Morris and McClatchey 2009; Dalgliesh et al. 2010). The continued development of relevant mouse models and further investigation of how the EGFR and Hippo signaling pathways converge, perhaps through Merlin, to regulate normal organ size will undoubtedly facilitate our understanding of how perturbation of the normal functions of these pathways leads to tumor development.

\section{Acknowledgments}

We apologize to our colleagues whose work could not be cited due to lack of space. We thank Jaleel Shujath for critical reading of the manuscript. The work in the Kissil laboratory is supported in part by NIH grant R01CA124495 and the American Cancer Society. C.Y. is a current recipient of the Young Investigator Award from the Children's Tumor Foundation.

\section{References}

Benhamouche S, Curto M, Saotome I, Gladden AB, Liu C-H, Giovannini M, McClatchey AI. 2010. Nf2/Merlin controls progenitor homeostasis and tumorigenesis in the liver. Genes Dev (this issue). doi: 10.1101/gad.1938710.

Berasain C, Garcia-Trevijano ER, Castillo J, Erroba E, Lee DC, Prieto J, Avila MA. 2005a. Amphiregulin: An early trigger of liver regeneration in mice. Gastroenterology 128: 424-432.

Berasain C, Garcia-Trevijano ER, Castillo J, Erroba E, Santamaria M, Lee DC, Prieto J, Avila MA. 2005b. Novel role for amphiregulin in protection from liver injury. I Biol Chem 280: 19012-19020.

Buckley AF, Burgart LJ, Sahai V, Kakar S. 2008. Epidermal growth factor receptor expression and gene copy number in conventional hepatocellular carcinoma. Am I Clin Pathol 129: 245-251.

Camargo FD, Gokhale S, Johnnidis JB, Fu D, Bell GW, Jaenisch R, Brummelkamp TR. 2007. YAP1 increases organ size and expands undifferentiated progenitor cells. Curr Biol 17: 2054-2060.

Cole BK, Curto M, Chan AW, McClatchey AI. 2008. Localization to the cortical cytoskeleton is necessary for Nf2/merlindependent epidermal growth factor receptor silencing. Mol Cell Biol 28: 1274-1284.

Curto M, McClatchey AI. 2008. Nf2/Merlin: A coordinator of receptor signalling and intercellular contact. Br J Cancer 98: 256-262.

Curto M, Cole BK, Lallemand D, Liu CH, McClatchey AI. 2007. Contact-dependent inhibition of EGFR signaling by Nf2/ Merlin. J Cell Biol 177: 893-903.

Dalgliesh GL, Furge K, Greenman C, Chen L, Bignell G, Butler A, Davies H, Edkins S, Hardy C, Latimer C, et al. 2010. Systematic sequencing of renal carcinoma reveals inactivation of histone modifying genes. Nature 463: 360-363.

Dong J, Feldmann G, Huang J, Wu S, Zhang N, Comerford SA, Gayyed MF, Anders RA, Maitra A, Pan D. 2007. Elucidation of a universal size-control mechanism in Drosophila and mammals. Cell 130: 1120-1133.

Giovannini M, Robanus-Maandag E, van der Valk M, NiwaKawakita M, Abramowski V, Goutebroze L, Woodruff JM, Berns A, Thomas G. 2000. Conditional biallelic Nf2 mutation in the mouse promotes manifestations of human neurofibromatosis type 2. Genes Dev 14: 1617-1630.

Hamaratoglu F, Willecke M, Kango-Singh M, Nolo R, Hyun E, Tao C, Jafar-Nejad H, Halder G. 2006. The tumour-suppressor 
genes NF2/Merlin and Expanded act through Hippo signalling to regulate cell proliferation and apoptosis. Nat Cell Biol 8: 27-36.

Ito Y, Takeda T, Sakon M, Tsujimoto M, Higashiyama S, Noda K, Miyoshi E, Monden M, Matsuura N. 2001a. Expression and clinical significance of erb-B receptor family in hepatocellular carcinoma. Br J Cancer 84: 1377-1383.

Ito $\mathrm{Y}$, Takeda T, Sasaki Y, Sakon M, Yamada T, Ishiguro S, Imaoka $S$, Tsujimoto $M$, Higashiyama $S$, Monden $M$, et al. 2001b. Expression and clinical significance of the erbB family in intrahepatic cholangiocellular carcinoma. Pathol Res Pract 197: 95-100.

Jang SW, Yang SJ, Srinivasan S, Ye K. 2007. Akt phosphorylates MstI and prevents its proteolytic activation, blocking FOXO3 phosphorylation and nuclear translocation. I Biol Chem 282: 30836-30844.

Jhappan C, Stahle C, Harkins RN, Fausto N, Smith GH, Merlino GT. 1990. TGF $\alpha$ overexpression in transgenic mice induces liver neoplasia and abnormal development of the mammary gland and pancreas. Cell 61: 1137-1146.

Keng VW, Villanueva A, Chiang DY, Dupuy AJ, Ryan BJ, Matise I, Silverstein KA, Sarver A, Starr TK, Akagi K, et al. 2009. A conditional transposon-based insertional mutagenesis screen for genes associated with mouse hepatocellular carcinoma. Nat Biotechnol 27: 264-274.

Lee KP, Lee JH, Kim TS, Kim TH, Park HD, Byun JS, Kim MC, Jeong WI, Calvisi DF, Kim JM, et al. 2010. The HippoSalvador pathway restrains hepatic oval cell proliferation, liver size, and liver tumorigenesis. Proc Natl Acad Sci 107: $8248-8253$.

Leone F, Cavalloni G, Pignochino Y, Sarotto I, Ferraris R, Piacibello W, Venesio T, Capussotti L, Risio M, Aglietta M. 2006. Somatic mutations of epidermal growth factor receptor in bile duct and gallbladder carcinoma. Clin Cancer Res 12: 1680-1685.

Lu L, Li Y, Kim SM, Bossuyt W, Liu P, Qiu Q, Wang Y, Halder G, Finegold MJ, Lee JS, et al. 2010. Hippo signaling is a potent in vivo growth and tumor suppressor pathway in the mammalian liver. Proc Natl Acad Sci 107: 1437-1442.

Lubner SI, Mahoney MR, Kolesar JL, Loconte NK, Kim GP, Pitot HC, Philip PA, Picus J, Yong WP, Horvath L, et al. 2010. Report of a multicenter phase II trial testing a combination of biweekly bevacizumab and daily erlotinib in patients with unresectable biliary cancer: A Phase II Consortium study. J Clin Oncol doi: 10.1200/JCO.2010.28.4075.

Lurje G, Lenz HJ. 2009. EGFR signaling and drug discovery. Oncology 77: 400-410.

Maitra S, Kulikauskas RM, Gavilan H, Fehon RG. 2006. The tumor suppressors Merlin and Expanded function cooperatively to modulate receptor endocytosis and signaling. Curr Biol 16: 702-709.

Matallanas D, Romano D, Yee K, Meissl K, Kucerova L, Piazzolla D, Baccarini M, Vass JK, Kolch W, O'Neill E. 2007. RASSF1A elicits apoptosis through an MST2 pathway directing proapoptotic transcription by the p73 tumor suppressor protein. Mol Cell 27: 962-975.

McClatchey AI, Saotome I, Ramesh V, Gusella JF, Jacks T. 1997. The Nf2 tumor suppressor gene product is essential for extraembryonic development immediately prior to gastrulation. Genes Dev 11: 1253-1265.

McClatchey AI, Saotome I, Mercer K, Crowley D, Gusella JF, Bronson RT, Jacks T. 1998. Mice heterozygous for a mutation at the Nf2 tumor suppressor locus develop a range of highly metastatic tumors. Genes Dev 12: 1121-1133.

Morris ZS, McClatchey AI. 2009. Aberrant epithelial morphology and persistent epidermal growth factor receptor signal- ing in a mouse model of renal carcinoma. Proc Natl Acad Sci 106: 9767-9772.

Nagy P, Bisgaard HC, Santoni-Rugiu E, Thorgeirsson SS. 1996. In vivo infusion of growth factors enhances the mitogenic response of rat hepatic ductal (oval) cells after administration of 2-acetylaminofluorene. Hepatology 23: 71-79.

Natarajan A, Wagner B, Sibilia M. 2007. The EGF receptor is required for efficient liver regeneration. Proc Natl Acad Sci 104: 17081-17086.

O'Neill E, Rushworth L, Baccarini M, Kolch W. 2004. Role of the kinase MST2 in suppression of apoptosis by the protooncogene product Raf-1. Science 306: 2267-2270.

Pineau P, Marchio A, Nagamori S, Seki S, Tiollais P, Dejean A. 2003. Homozygous deletion scanning in hepatobiliary tumor cell lines reveals alternative pathways for liver carcinogenesis. Hepatology 37: 852-861.

Romano D, Matallanas D, Weitsman G, Preisinger C, Ng T, Kolch W. 2010. Proapoptotic kinase MST2 coordinates signaling crosstalk between RASSF1A, Raf-1, and Akt. Cancer Res 70: 1195-1203.

Sandgren EP, Luetteke NC, Palmiter RD, Brinster RL, Lee DC. 1990. Overexpression of TGF $\alpha$ in transgenic mice: Induction of epithelial hyperplasia, pancreatic metaplasia, and carcinoma of the breast. Cell 61: 1121-1135.

Scoles DR. 2008. The merlin interacting proteins reveal multiple targets for NF2 therapy. Biochim Biophys Acta 1785: 3254.

Song H, Mak KK, Topol L, Yun K, Hu J, Garrett L, Chen Y, Park O, Chang J, Simpson RM, et al. 2010. Mammalian Mst1 and Mst2 kinases play essential roles in organ size control and tumor suppression. Proc Natl Acad Sci 107: 1431-1436.

Striedinger $\mathrm{K}$, VandenBerg SR, Baia GS, McDermott MW, Gutmann DH, Lal A. 2008. The neurofibromatosis 2 tumor suppressor gene product, merlin, regulates human meningioma cell growth by signaling through YAP. Neoplasia 10: 1204-1212.

Thomas MB, Morris JS, Chadha R, Iwasaki M, Kaur H, Lin E, Kaseb A, Glover K, Davila M, Abbruzzese J. 2009. Phase II trial of the combination of bevacizumab and erlotinib in patients who have advanced hepatocellular carcinoma. J Clin Oncol 27: 843-850.

$\mathrm{Xu}$ MZ, Yao TJ, Lee NP, Ng IO, Chan YT, Zender L, Lowe SW, Poon RT, Luk JM. 2009. Yes-associated protein is an independent prognostic marker in hepatocellular carcinoma. Cancer 115: 4576-4585.

Yokoyama T, Osada H, Murakami H, Tatematsu Y, Taniguchi T, Kondo Y, Yatabe Y, Hasegawa Y, Shimokata K, Horio Y, et al. 2008. YAP1 is involved in mesothelioma development and negatively regulated by merlin through phosphorylation. Carcinogenesis 29: 2139-2146.

Yu J, Zheng Y, Dong J, Klusza S, Deng WM, Pan D. 2010. Kibra functions as a tumor suppressor protein that regulates Hippo signaling in conjunction with Merlin and Expanded. Dev Cell 18: 288-299.

Yuan Z, Kim D, Shu S, Wu J, Guo J, Xiao L, Kaneko S, Coppola D, Cheng JQ. 2010. Phosphoinositide 3-kinase/Akt inhibits MST1-mediated pro-apoptotic signaling through phosphorylation of threonine 120. J Biol Chem 285: 3815-3824.

Zender L, Spector MS, Xue W, Flemming P, Cordon-Cardo C, Silke J, Fan ST, Luk JM, Wigler M, Hannon GJ, et al. 2006. Identification and validation of oncogenes in liver cancer using an integrative oncogenomic approach. Cell 125: 12531267.

Zhang J, Ji JY, Yu M, Overholtzer M, Smolen GA, Wang R, Brugge JS, Dyson NJ, Haber DA. 2009. YAP-dependent induction of amphiregulin identifies a non-cell-autonomous 
component of the Hippo pathway. Nat Cell Biol 11: 14441450.

Zhao B, Wei X, Li W, Udan RS, Yang Q, Kim J, Xie J, Ikenoue T, $\mathrm{Yu}$ J, Li L, et al. 2007. Inactivation of YAP oncoprotein by the Hippo pathway is involved in cell contact inhibition and tissue growth control. Genes Dev 21: 2747-2761.

Zhao B, Li L, Lei Q, Guan KL. 2010. The Hippo-YAP pathway in organ size control and tumorigenesis: An updated version. Genes Dev 24: 862-874.

Zhou D, Conrad C, Xia F, Park JS, Payer B, Yin Y, Lauwers GY, Thasler W, Lee JT, Avruch J, et al. 2009. Mst1 and Mst2 maintain hepatocyte quiescence and suppress hepatocellular carcinoma development through inactivation of the Yap1 oncogene. Cancer Cell 16: 425-438. 


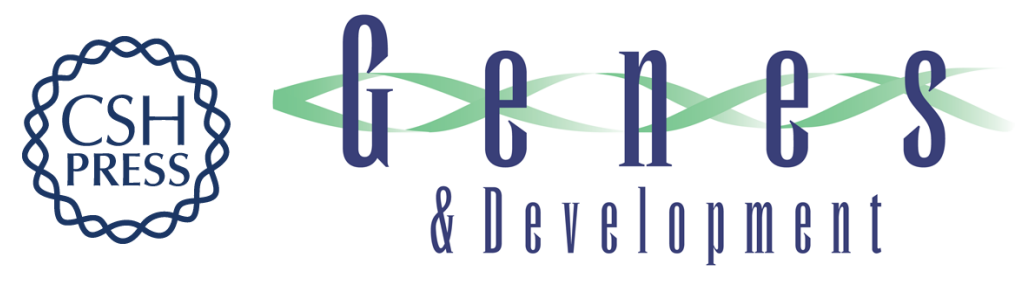

\section{Merlin in organ size control and tumorigenesis: Hippo versus EGFR?}

Chunling Yi and Joseph L. Kissil

Genes Dev. 2010, 24:

Access the most recent version at doi:10.1101/gad.1964810

Related Content Nf2/Merlin controls progenitor homeostasis and tumorigenesis in the liver Samira Benhamouche, Marcello Curto, Ichiko Saotome, et al.

Genes Dev. August , 2010 24: 1718-1730

References This article cites 44 articles, 19 of which can be accessed free at:

http://genesdev.cshlp.org/content/24/16/1673.full.html\#ref-list-1

Articles cited in:

http://genesdev.cshlp.org/content/24/16/1673.full.html\#related-urls

\section{License}

Email Alerting

Receive free email alerts when new articles cite this article - sign up in the box at the top Service right corner of the article or click here.

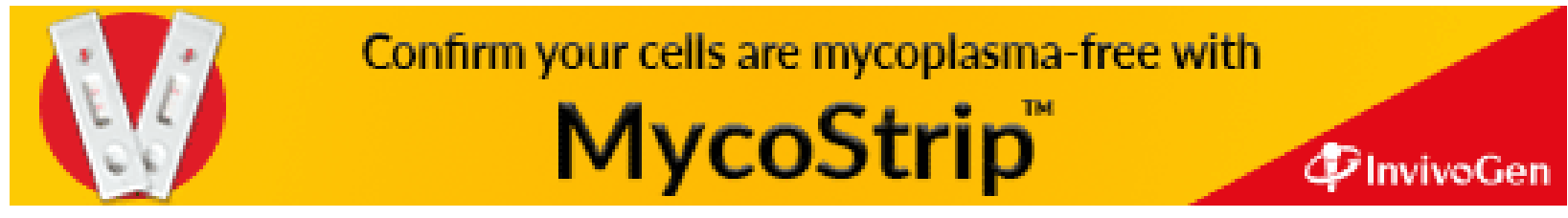

